\title{
Evaluate the Effect of Coenzymeq10 Supplementation on Reducing Oxidative Stress in Patients with Relapsing Remitting Multiple Sclerosis
}

\author{
Mariam OmranWaheed ${ }^{1}$, Haider Al- Tukmagi ${ }^{2}$, Ali Kadhim Kareem ${ }^{3}$ \\ ${ }^{1}$ Department of Clinical Pharmacy, Pharmacy College, Adiwanyaa general hospital,Qadisea, Iraq \\ ${ }^{2}$ Department of Clinical Pharmacy, Pharmacy College, Baghdad University, Baghdad, Iraq \\ ${ }^{3}$ Department of Medicine, Baghdad Teaching Hospital, Baghdad, Iraq
}

\begin{abstract}
Background: Multiple sclerosis it's a disease cause demyelination of the central nervous system (CNS). it's long duration result in individual, family tremendous \& societal cost as well as reduction in life quality \& work productivity. oxidative stress may play a key role in its pathogenesis. The aim of this study was to evaluate the effect of coenzyme Q10 (CoQ10) 200mg/day supplementation on oxidative stress and antioxidant enzyme activity in patients with relapsing-remitting MS (RRMS). Materials and Methods: the study was a single-blind, randomized (placebo-controlled) study to determine the effect of CoQ10 supplement (200 mg/day, $n=20)$ versus placebo (control, $n=20)$ for 24 weeks. Blood samples were taken before and after a 24-week intervention to analyze malondialdehyde (MDA) and the antioxidant enzyme superoxide dismutase (SOD) activity, liver and renal function (aspartate aminotransferase (AST), alanine aminotransferase (ALT), alkaline phosphatase (AlkP), s. urea and s. creatinine) was measured to evaluate the safety of CoQ10. Results: Forty subjects with RRMS completed intervention study. After 24 weeks, CoQ10-treated patients had significant increase in SOD activity $(p=0.000)$; and decrease in MDA levels $(P=0.000)$ compared with placebo group. CoQ10 supplementation have no significant effect on EDSS, liver and renal function. Conclusion: in the present study suggests that CoQ10 supplements at a dose of $200 \mathrm{mg} / \mathrm{day}$ for 24 weeksis safe and can decrease oxidative stress and increase antioxidant enzyme activity in patients with RRMS.
\end{abstract}

Keywords: Coenzyme Q10, Oxidative stress, Relapsing remitting MS patients

\section{Introduction}

Multiple sclerosis it's a disease cause demyelination of the central nervous system $(\mathrm{CNS})^{[1]}$. The diagnosis required the occurrence of at least 2 neurological events that consist with CNS demyelination which separate temporally \& anatomically. The onset of the disease early in life (occur typically between the age of 20 \& 40 years) \& it's long duration result in individual, family tremendous $\&$ societal cost as well as reduction in life quality \& productivity in work $^{[2]}$. Multiple sclerosis (MS) has characteristic, variable $\&$ unpredictable course, the symptoms could include visual loss, weakness, bowel \& bladder incontinence, cognitive impairment, fatigue \&mood symptoms.

2.5 million people in worldwide affected with MS approximately, four hundred thousand of people in United States, and 200 people and more to have diagnose with MS each week in the United States ${ }^{[3]}$. MS typically begins 20 to 40 years of age. In young adults it is consider the leading cause of non-traumatic disability. Initial symptom are rarely occur before the age 10 years or after the age of $60 \mathrm{yrs}^{[4]}$. It affect women twice as often as men.

The true extent and nature of MS pathology within and between patients, and over time, remains unclear ${ }^{[5]}$. There is substantial evidence, however, to suggest it is a process of autoimmune that directed against oligodendrocytes (the cells that make CNS myelin) and myelin ${ }^{[6]}$. The mediator that actually cause destruction of myelin \& axonal hasn't been established, but it may reflects a combination of macrophage, reactive oxygen \& intermediate of destructive cytokines. The clinical findings of MS are not unique, but the disease have some findings that are highly characteristic.

pathogenic evidence suggests a possible role for oxidative stress $(\mathrm{OS})$ in the pathogenesis of $\mathrm{MS}^{[7]}$ Several studies indicate elevation of some markers of oxidation in cerebrospinal fluid and serum of patients with $\mathrm{MS}^{[8]}$. Reactive oxygen species ROS which is a small molecule that are highly reactive, due to presence of unpaired electron or their ability to give rise to new free radical production, that contributes to cellular injury ${ }^{[9]}$. Although, there are protective system against free radicals, these systems responses are overwhelmsin pathological conditions like MS. This condition leads to the employment of many of antioxidants in order to enhance MS.

Experimental animal studies have demonstrated that dietary intake of exogenous antioxidants, reduce the progression and clinical signs of (experimental autoimmune encephalomyelitis) $\mathrm{EAE}^{(9)}$. Recently CoQ10 is used as a neuroprotective agent in the treatment of different neurodegenerative impairments such as Parkinson's disease (PD), Huntington's disease, amyotrophic lateral sclerosis(ALS) \&Friedreich's ataxia ${ }^{[10]}$. Several studies have investigate CoQ10 role against ROS damage and apoptotic cell death ${ }^{(11)}$. In this study a try was made to evaluate CoQ10 effect and safety in RRMS patients. 


\section{International Journal of Science and Research (IJSR) \\ ISSN (Online): 2319-7064}

Index Copernicus Value (2013): 6.14 | Impact Factor (2014): 5.611

\section{Subject and Material}

\subsection{Patient Selection}

40 patients (20 female and 20 male) with diagnosed MS wereenrolled in this study in addition to 20 Healthyvolunteers were served as control. Exclusion of smoking and alcohol drinking individuals, as well as individuals suffering from chronic diseases or any condition that may affect oxidative stress. The forty patients were divided into two groups randomly one group take CoQ10 (200)mg daily, the second group take placebo. Patients were evaluated at baseline and 24 weeks later. Patients were instructed to sustain their schedules of treatment.

\subsection{Sample Collection}

Venous blood was obtained for each individual (control and patient) by vein puncture before the initiation of therapy, as a baseline, and 24 weeks later. Estimation of Malondialdehyde (MDA)using (ELISA) kit apply the competitive enzymes immunoassay techniques. And superoxide dismutase (SOD) assessment by quantitative sandwich enzyme immune assay by (ELISA) kit to evaluate antioxidant activity of CoQ10,aspartate aminotransferase (AST), alanine aminotransferase (ALT), alkaline phosphatase (ALKP), serum urea and serum creatinine were determined by quantitation method to evaluate the safety of CoQ10. Expanded disability status scale (EDSS) has been evaluated before and after treatment.

\subsection{Statistical Analysis}

Statistical analysis performed by using SPSS-21 (Statistical Package for Social Sciences_V 21). Results are Means \pm $\mathrm{SE}$, statistical analysis wasperformed by one - way analysis of variance(ANOVA).P $<0.05$ was considered statistically significant $^{[13]}$.

\section{Result}

Of Fifty patients presented to the study only forty patients (20 male and 20 female) were complete the study. Their age were $35.65 \pm 9.294$ years (as mean $\pm \mathrm{SE}$ ). There were no apparent differences between the three groups with respect to demographic data (Table 1).At baseline there is no significant difference between Co Q10, placebo group in superoxide dusmitase (SOD)level in compare with control, while malondialdehyde (MDA) was in RRMS patients differ significantly $(\mathrm{P}>0.05)$ in compare to control . However, after 24 weeks of treatment (MDA) was significantly decrease in CoQ10 group $(\mathrm{p}<0.05)$ compared to the baseline value, with significant difference between CoQ10 group and placebo.

Table (1) Demographic data

\begin{tabular}{|c|c|c|c|c|}
\hline Parameter & $\begin{array}{c}\text { CoQ10 } \\
\text { group }\end{array}$ & $\begin{array}{c}\text { Placebo } \\
\text { group }\end{array}$ & $\begin{array}{c}\text { Control } \\
\text { group }\end{array}$ & P-value \\
\hline Age & $34.60+8.66$ & $35.65+2.10$ & $32.40+1.98$ & $\mathrm{NS}$ \\
\hline $\begin{array}{c}\text { Female: Male } \\
\text { (Female \%) }\end{array}$ & $10: 10(50 \%)$ & $10: 10(50 \%)$ & $10: 10(50 \%)$ & $\mathrm{NS}$ \\
\hline BMI & $24.29+0.46$ & $26.54+1.26$ & $24.90+0.88$ & $\mathrm{NS}$ \\
\hline
\end{tabular}

Data expressed as mean $\pm \mathrm{SE},{ }^{*} \mathrm{BMI}=$ body mass index, which is calculated according to the following formula: $\mathrm{BMI}=$ weight (kilogram)/hight2 (meter), NS= non-significant $(\mathrm{p}>0.05)$.

Level of SOD was significantly increased in CoQ10 group from baseline with significant difference between CoQ10 and placebo.

Serum AST, ALT, AlkP, urea and creatinine have no significant difference between CoQ10, placebo and control group at baseline. After treatment there is no significant difference in compare to baseline, furthermore there was no significant difference between CoQ10 and placebo.

There was no significant differences in EDSSat baseline between CoQ10 group and placebo. However, there was no significant change among CoQ10 group after 24 weeks of treatment.

\section{Discussion}

Co Q10 involved in energy production $41^{[14]}$, and acts as a primary scavenger of free radicals, protecting from oxidative damage and favoring the regeneration of other antioxidants ${ }^{[15]} .42$ It has anti-inflammatory properties because it inhibits metalloproteinases and IL-6 production $^{[16]}$. In this study a supplementation of $200 \mathrm{mg}$ of CoQ10 over 24 wks resulted in a significant decrease $(p>0.05)$ in MDA level compared with placebo in group of RRMS patients, this result is in constant with the result of MeisamSanoobar and colleagues ${ }^{[17]}$ who find that MDA level was reduced in RRMS patients taking supplement of CoQ10 (500 mg once daily)over 12 wks.

Level of SOD in this study was significantly increased $(p>0.05)$ compare to placebo, in a study by Lee et al. in the same line ${ }^{[18]}$, activity of SOD increased in patient with coronary artery disease (CAD) after 12-week with CoQ10 supplementation. EDSS value show no significant change in CoQ10 group in compare to placebo this result is in an agreement with MairaGironi et al., who suggest that antioxidant depletion as an intrinsic, probably a causative factor associated with MS course rather than a consequence $^{[19]}$.

Monitoring of liver and renal functions showed the safety of use of CoQ10 cap. in RRMS patient. Comparing the measured levels of serum ALT, AST, AlpK, urea and creatinine between CoQ10 and placebo groups show no significant differences over 24 wksof treatment.

\section{Conclusion}

Administration of coenzyme Q10 (200mg/day) decrease lipid peroxidation and increase antioxidant enzyme activity and was safe in patients with MS, CoQ10 by its antioxidant activity, Enhanced antioxidant mechanisms may represent a natural compensatory mechanism protecting against direct oxidative damage. The antioxidant activity can be considered as one of the main factors responsible for the neuroprotective effect of CoQ10. Further larger and longer studies are warranted to confirm these findings. 


\section{International Journal of Science and Research (IJSR) \\ ISSN (Online): 2319-7064 \\ Index Copernicus Value (2013): 6.14 | Impact Factor (2014): 5.611}

\section{Acknowledgement}

The present work was abstracted from MSc theses submitted to the Department of Clinical Pharmacy, College of Pharmacy, and University of Baghdad. The authors gratefully thank Consultant clinic of multiple sclerosisin Baghdad Teaching Hospital/ Medical city/Baghdad for supporting the project.

\section{References}

[1] Anderson DW, Ellenberg $\mathrm{JH}$, Leventhal CM, et al. Revised estimate of the prevalence of multiple sclerosis in the United States. Ann Neuro 1992;31:333- 336.

[2] Sadovnick AD, Ebers GC. Epidemiology of multiple sclerosis: A critical overview. Can J NeurolSci 1993;20:17-29.

[3] National Multiple Sclerosis Society. About MS: what we know about MS. http://www.nationalmssociety.org/aboutmultiplesclerosis/what-we-know- about-ms/index.aspx. Accessed October 20, 2012.

[4] Dr.J Palace, "Making the diagnosis of multiple sclerosis" Journal of Neurology, Neurosurgery and Psychiatry,vol.71,2004

[5] Sobel RA. The pathology of multiple sclerosis. NeurolClin 1995;13:1-16.

[6] Trapp BD, Peterson J, Ransohoff RM, et al. Axonal transection in the lesions of multiple sclerosis. N Engl J Med 1998;338:278-285.

[7] Truyen L, van Wuesberghe JHTM, Barkof F, et al. Accumulation of hypointense lesions ("black holes") on T1 spin echo MRI correlates with disease progression in multiple sclerosis. Neurology 1996;47:1469-1476.

[8] Hartung HP, Kieseier BC. The role of matrix metalloproteinases in autoimmune damage to the central and peripheral nervous system. J Neuroimmunol 2000;107: 140/47.

[9] Van Horssen, Jack, et al. "Radical changes in multiple sclerosis pathogenesis." Biochimica et BiophysicaActa (BBA)-Molecular Basis of Disease 1812.2 (2011): 141150
[10] Sadowska-Bartosz, I., Adamczyk-Sowa, M., Galiniak, S., Mucha, S., Pierzchala, K. and Bartosz, G. (2013) Oxidative modification of serum proteins in multiple sclerosis. Neurochemisty International, 63, 507-516.

[11]Besler, H.T. and Comoglu, S. (2003) Lipoprotein oxidation, plasma total antioxidant capacity and homocysteine level in patients with multiple sclerosis. Nutritional Neuroscience, 6, 189-196.

[12] McCarthy S, Somayajulu M, Sikorska M, BorowyBorowski H, Pandey S. Paraquat induces oxidative stress and neuronal cell death; neuroprotection by water-soluble coenzyme Q10. ToxicolApplPharmacol 2004; 201: 21-31.

[13] SPSS.IBM Corp. Released 2012. IBM SPSS Statistics for Windows, Version 21.0. Armonk, NY: IBM Corp.

[14] G. P. Littarru and L. Tiano, "Clinical aspects of coenzyme Q10: an update," Nutrition, vol. 26, no. 3, pp. 250-254, 2010. View at Publisher •

[15]D. A. Groneberg, B. Kindermann, M. Althammer et al., "Coenzyme Q10 affects expression of genes involved in cell signalling, metabolism and transport in human CaCo-2 cells," International Journal of Biochemistry and Cell Biology, vol. 37, no. 6, pp. 1208-1218, 2005.

[16] M. Inui, M. Ooe, K. Fujii, H. Matsunaka, M. Yoshida, and M. Ichihashi, "Mechanisms of inhibitory effects of CoQ10 on UVB-induced wrinkle formation in vitro and in vivo," BioFactors, vol. 32, no. 1-4, pp. 237-243, 2008.

[17] M. Sanoobar, S. Eghtesadi, A. Azimi, et al., "Coenzyme Q10 supplementation reduces oxidative stress and increases antioxidant enzyme activity in patients with relapsing-remitting multiple sclerosis," International Journal of Neuroscience, vol. 123, pp. 776-782, 2013.

[18] Lee BJ, Lin YC, Huang YC, The relationship between coenzyme Q10, oxidative stress, and antioxidant enzymes activities and coronary artery disease. Sci World J 2012;2012:1-8.

[19] MairaGironi, Bruno Borgiani, EnricaMariani, et al., "Oxidative Stress Is Differentially Present in Multiple Sclerosis Courses, Early Evident, and Unrelated to Treatment," Journal of Immunology Research, vol. 2014, Article ID 961863, 9 pages, 2014. doi:10.1155/2014/961863

Table 2: Changes from baseline and after 24weeks in laboratory parameters for patients who completed the study

\begin{tabular}{|c|c|c|c|}
\hline Parameter & $\begin{array}{c}\text { Coenzyme 10 } \\
\text { group 20 subjects }\end{array}$ & $\begin{array}{c}\text { Placebo group } \\
20 \text { subjects }\end{array}$ & $\begin{array}{c}\text { Control group } \\
20 \text { subjects }\end{array}$ \\
\hline SOD(pg/ml) Before intervention After intervention & $271.86 \pm 29.46$ & $363.99 \pm 51.62$ & $464.71 \pm 23.66$ \\
& $420.66 \pm 34.21^{*}$ & $249.63 \pm 28.64^{*}$ & \\
\hline MDA (ng/ml) Before intervention After intervention & $4.38 \pm 0.16^{* *}$ & $4.00 \pm 0.10^{* *}$ & $3.38 \pm 0.19$ \\
& $3.32 \pm 0.18^{*}$ & $4.53 \pm 0.16^{*}$ & \\
\hline ALT(U/L) Before intervention After intervention & $30.00 \pm 3.97$ & $26.95 \pm 4.11$ & $19.8 \pm 2.49$ \\
& $33.80 \pm 5.72$ & $30.35 \pm 1.81$ & \\
\hline AST(U/L)Before intervention After intervention & $28.45 \pm 2.87$ & $29.25 \pm 2.72$ & $20.60 \pm 1.08$ \\
\hline AlkP(U/L)Before intervention After intervention & $32.05 \pm 6.51$ & $26.25 \pm 1.93$ & \\
& $75.20 \pm 5.23$ & $89.35 \pm 4.75$ & $71.30 \pm 4.29$ \\
\hline Urea (mg/dL)Before intervention After intervention & $26.15 \pm 1.47$ & $28.80 \pm 1.97$ & $27.30 \pm 2.25$ \\
\hline Cr(mg/dL) Before intervention After intervention & $24.20 \pm 1.24$ & $28.15 \pm 1.33$ & \\
\hline
\end{tabular}

Data represented as mean $\pm \mathrm{SE}$ for baseline and end of study values; ${ }^{*}$ significantly different when compared to pre-treatment level within the same group $(\mathrm{P}<0.05)$;** significantly different when compared to control group $(\mathrm{p}<0.05)$. 